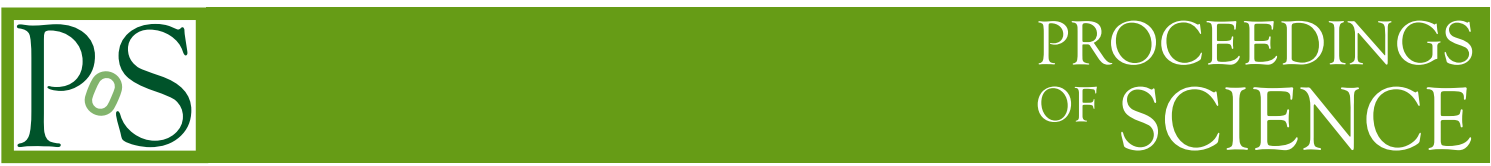

\title{
The new $p$-process database of KADoNiS
}

\author{
T. Szücs*广 \\ Institute of Nuclear Research (ATOMKI), Debrecen, Hungary \\ E-mail: tszucs@atomki.hu
}

\author{
I. Dillmann \\ GSI Helmholtzzentrum für Schwerionenforschung GmbH, Darmstadt, Germany \\ II. Physikalisches Institut, Justus-Liebig-Universität, Giessen, Germany

\section{R. Plag ${ }^{\S}$} \\ GSI Helmholtzzentrum für Schwerionenforschung GmbH, Darmstadt, Germany \\ Goethe-Universität, Frankfurt am Main, Germany
}

\section{Zs. Fülöp ${ }^{\mathbb{I}}$}

Institute of Nuclear Research (ATOMKI), Debrecen, Hungary

\begin{abstract}
The KADoNiS (Karlsruhe Astrophysical Database of Nucleosynthesis in Stars) project is an online database (www.kadonis.org) for cross sections relevant to the $s$-process and the $p$-process. The first version was an updated sequel to the previous Bao et al. [1] compilations from 1987 and 2000 for $(n, \gamma)$ cross sections relevant to Big Bang and $s$-process nucleosynthesis. The first update, KADoNiS v0.2, was published in 2006 [2]. It contained mainly Maxwellian averaged $(n, \gamma)$ cross sections relevant to the $s$-process, and some experimental charged particle induced reaction relevant to the $p$-process. After that a second update was presented in 2009 [3].

Recently, we started to collect and review all existing experimental data relevant for $p$-process nucleosynthesis and to provide a user-friendly database based on the KADoNiS framework. The $p$-process part of the KADoNiS database is currently being extended and will include all available experimental data from $(p, \gamma),(p, n),(p, \alpha),(\alpha, \gamma),(\alpha, n)$ and $(\alpha, p)$ reactions in or close to the respective Gamow window.
\end{abstract}

11th Symposium on Nuclei in the Cosmos, NIC XI

July 19-23, 2010

Heidelberg, Germany

\footnotetext{
*Speaker.

${ }^{\dagger}$ T.Sz. is supported by EUROGENESIS OTKA-NN83261.

$\ddagger$ I.D. is supported by the Helmholtz society via the Young Investigators project VH-NG-627.

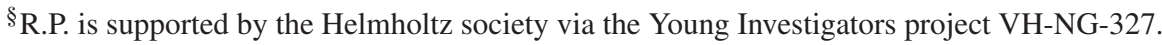

I Zs.F. is supported by OTKA K68809.
} 


\section{Introduction}

The nuclei heavier than iron are produced by the three processes called $s-, r$-, and $p$-process [4]. The first two involve slow and rapid neutron capture reactions and $\beta$-decays. In the $s$-process the time scale of the neutron capture is much longer than the $\beta$-decay half-lives, therefore the reaction flow is running close to the valley of stability up to ${ }^{209} \mathrm{Bi}$. The two sites of the $s$-process are low mass AGB stars for the main $s$-process [5] and massive stars for the weak $s$-process [6].

In the $r$-process the neutron density is so high, that subsequent neutron captures drive the material far away from the line of stability close to the neutron drip line. When the neutron density drops and the temperature decreases ("freeze out"), the material decays back to the stability via long $\beta$-decay chains. Such a high neutron density requires an explosive stellar environment. The exact $r$-process scenario is still under discussion, but the presently favoured candidates are core collapse supernovae [7] and merging neutron stars [8].

There are about 30 isotopes (less than $1 \%$ of the total solar abundance beyond iron) on the proton-rich side of the valley of stability, which cannot be produced via neutron capture reactions. The main fraction of these so-called $p$-nuclei is produced in the $\gamma$-process [9]. It proceeds by photon-induced reactions starting from pre-existing $s$ - or $r$-process seed nuclei. First $(\gamma, n)$ reactions drive the material towards the neutron deficient region, before charged-particle emitting reactions like $(\gamma, \alpha)$ and $(\gamma, \mathrm{p})$ become possible and divert the reaction flow towards lower masses.

However, astrophysical network calculations cannot fully reproduce the observed abundances of all $p$-nuclei within one scenario and call for additional processes [10]. These reaction network calculations involve thousands of stable and unstable isotopes and tens of thousands of reactions [11]. Only the minority of these reactions is known experimentally, whereas the cross sections for the largest fraction have to be inferred from statistical model calculations.

The fact that self-consistent studies of the $\gamma$-process have problems in synthesizing $p$-nuclei in the mass regions $A<124$ and $150<A<165$ may result from difficulties related to the astrophysical models as well as from systematic uncertainties of the nuclear physics input. Therefore, an improvement of nuclear reaction cross sections is crucial for further progress in $p$-process models, either by directly replacing theoretical predictions by experimental data or by testing and improving the reliability of statistical models, if the relevant energy range (the so called Gamow window) is not accessible by experiments.

\section{The new $p$-process database}

The main aim of the new $p$-process database is the collection of all available experimental cross sections in or close to the respective Gamow window and the comparison with various theoretical models. An important constraint is also - like in the s-process part of KADoNiS - the user-friendliness and easy access to the data. This database is not only aimed at stellar modellers, but also for experimentalists who want to get a quick overview about the experimental situation.

The basis of the new p-process database was the EXFOR database [12], which has the advantage that it contains (almost) all available experimental cross sections and is regularly updated. However, EXFOR contains also a lot of irrelevant data for the $p$-process, because most measurements were performed far away from the Gamow window and have no astrophysical im- 
portance. Another difficulty arose from the non-uniformity of the datasets. In EXFOR either the center-of-mass energy was used, or the energy in laboratory system, depending on the original publication. The error handling is also not done uniformly, in some datasets global errors, in others individual errors were given, or even different units were used. And just few of the datasets contain an astrophysical $S$-factor, however this quantity is needed for the extrapolations.

Therefore we scanned the EXFOR database for experimental data from $(p, \gamma),(p, n),(p, \alpha)$, $(\alpha, \gamma),(\alpha, n)$, and $(\alpha, p)$ reactions for targets heavier than ${ }^{70} \mathrm{Ge}$ and energies down to $1.5 \times$ the upper end of the respective Gamow window for $T=3 \mathrm{GK}$. This upper cut-off energy was arbitrarily chosen because most available data was measured above the astrophysically important Gamow windows, which lie (for $T=3 \mathrm{GK}$ ) between $E=3$ and $6 \mathrm{MeV}$ for proton-induced and between 7 and $14 \mathrm{MeV}$ for $\alpha$-induced reactions.

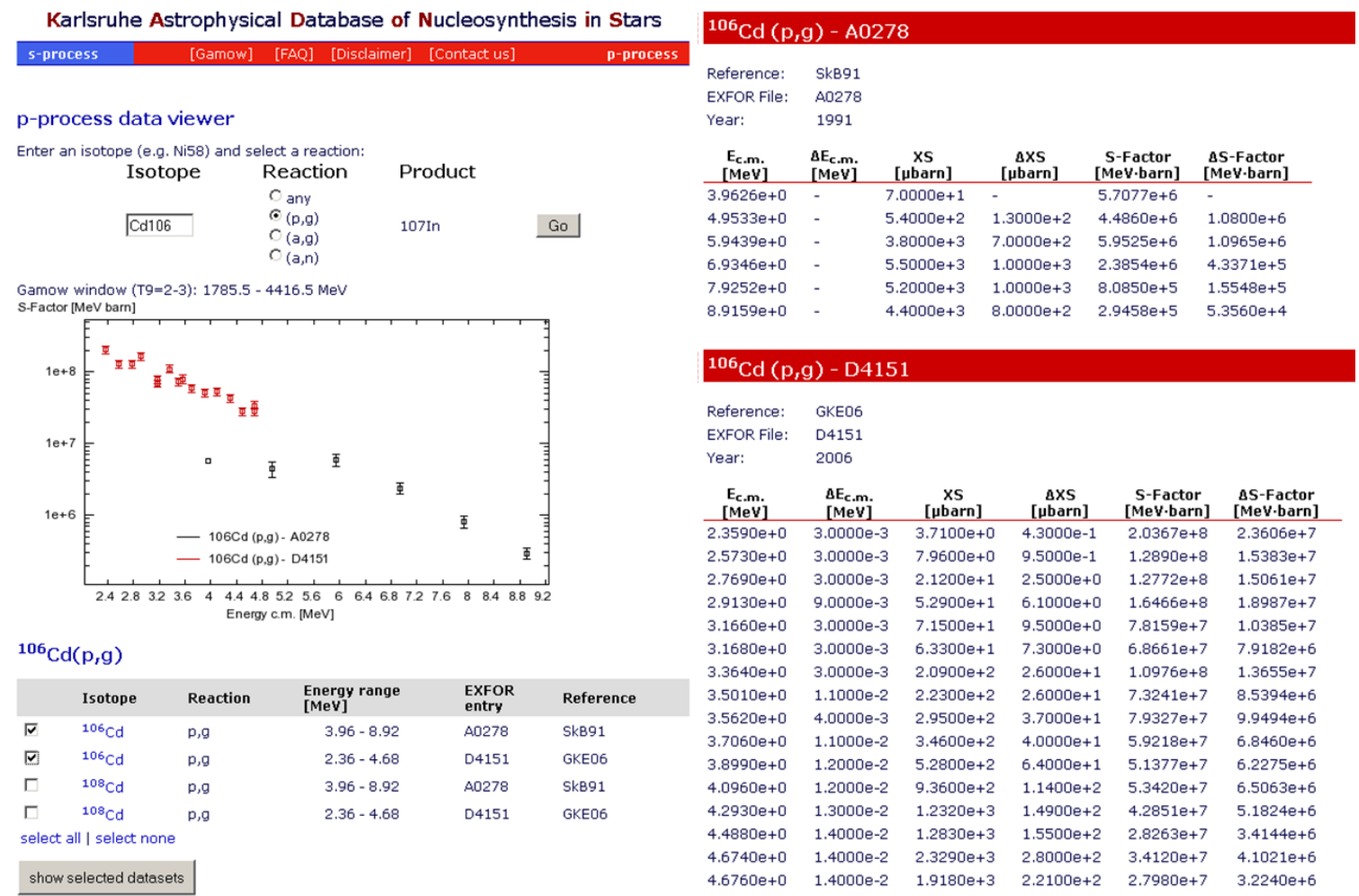

Figure 1: Screenshots from the online plotter from the datasheet of ${ }^{106} \mathrm{Cd}(p, \gamma)$.

In Fig. 1 screenshots from the KADoNiS p-process database are shown. After selection of the isotope and the reaction, the datasheet provides the calculated Gamow window between $T=2$ and $3 \mathrm{GK}$ for the respective reaction from [13]. One can also select one or more datasets and plot these with an online plotter, and show the data in tabulated form. In this table the center-of-mass energy of the reaction and the uncertainty of this energy (if available) are given or were calculated if only laboratory energies were provided in the original publication. In the next columns are the cross sections and experimental uncertainties (if available), and then an astrophysical $S$-factor is calculated.

The experimental basis for $(p, \gamma),(p, n),(p, \alpha),(\alpha, \gamma),(\alpha, n)$, and $(\alpha, p)$ cross sections is very 
different with respect to the energy window for $p$-process nucleosynthesis. No $(p, \alpha)$ cross section exists within our defined upper cut-off energy, whereas for the inverse $(\alpha, p)$ channel eight reactions are available $\left({ }^{92,94} \mathrm{Zr},{ }^{92,98} \mathrm{Mo},{ }^{96} \mathrm{Ru},{ }^{106} \mathrm{Cd}\right.$, and $\left.{ }^{112,124} \mathrm{Sn}\right)$. However, both reactions play no or only a negligible role in present $\gamma$-process models. The most information over the whole mass region can be found for $(\alpha, n)$ and $(p, n)$ reactions. A lot of effort with respect to astrophysical measurements in the last 15 years was put in determining radiative capture cross sections, which can be related to the photo-induced reactions by detailed balance. For $(p, \gamma)$ reactions up to now 34 datasets are available, most of them in the lower mass region where their influence was found to be largest [14].

The situation for $(\alpha, \gamma)$ is much worse, and it was stated by many authors before that more effort should be put in measuring this type of reaction with heavier targets. Up to today only 16 isotopes have been measured (and published): ${ }^{70} \mathrm{Ge},{ }^{91} \mathrm{Zr},{ }^{96} \mathrm{Ru},{ }^{106} \mathrm{Cd},{ }^{107} \mathrm{Ag},{ }^{113,115} \mathrm{In},{ }^{112,117,118} \mathrm{Sn},{ }^{127} \mathrm{I}$, ${ }^{136} \mathrm{Xe},{ }^{139} \mathrm{La},{ }^{144,154} \mathrm{Sm}$, and ${ }^{197} \mathrm{Au}$. All of these measurements do not reach the lower end of the Gamow window.

\section{Further plans}

One of our next plans is give recommended reaction rates for each reaction and derive fit parameters, which can easily be included in reaction libraries. We will implement theoretical data from the literature as a comparison to the recommended reaction rates, which helps to compare the reliability of different theoretical calculations. For 2011 we then plan a publication of the KADoNiS p-process database.

\section{References}

[1] Z. Y. Bao, H. Beer, F.Käppeler, F. Voss, K. Wisshak, and T. Rauscher, Atomic Data and Nuclear Data Tables 76, 70 (2000)

[2] I. Dillmann, M. Heil, F. Käppeler, R. Plag, T. Rauscher, and F.-K. Thielemann, AIP Conf. Proc. 819, 123 (2006)

[3] I. Dillmann, R. Plag, F. Käppeler, T. Rauscher, EFNUDAT Fast Neutrons, Proceedings of the "Scientific Workshop on Neutron Measurements, Theory and Applications", April 2009, Geel/Belgium, edt. F.-J. Hambsch, p. 55 (2010)

[4] M. E. Burbidge, G.R. Burbidge, W.A. Fowler, and F. Hoyle, Rev. Mod. Phys. 29, 547 (1957)

[5] M. Busso, R. Gallino, and G. J. Wasserburg, Annu. Rev. Astron. Astrophys. 37, 239 (1999)

[6] L.-S. The, M. El Eid, and B. Meyer, Astrophys. J. 533, 998 (2000)

[7] Y.-Z. Qian, Astrophys. J. Lett. 534, L67 (2000)

[8] C. Freiburghaus, S. Rosswog, and F.-K. Thielemann, Astrophys. J. 525, L121 (1999)

[9] S.E. Woosley, W.M. Howard Ap. J. Suppl. 36, 285 (1978)

[10] M. Arnould and S. Goriely, Phys. Rep. 384, 1 (2003)

[11] M. Rayet, et al., Astron. Astroph. 227, 271 (1990)

[12] http://www-nds.iaea.org/exfor/exfor.htm

[13] T. Rauscher, Phys Rev. C 81, 045807 (2010)

[14] W. Rapp, J. Görres, M. Wiescher, H. Schatz, and F. Käppeler, Ap. J. 653, 474 (2006). 